\title{
Are low dose pioglitazone combinations justifiable?
}

\author{
Rakesh M. Parikh
}

Received: 28 March 2011 / Accepted: 23 August 2011 /Published online: 20 September 2011

(C) Research Society for Study of Diabetes in India 2011

Dear Sir,

Recently number of pharmaceutical companies in India have started marketing various oral hypoglycemic agent (OHA) combinations with low dose $(7.5 \mathrm{mg})$ pioglitazone. All these combinations are based on very small data from two studies conducted in the Japanese population. Based on these studies, low dose pioglitazone is promoted to be having efficacy comparable to that of $15 \mathrm{mg}$ with significantly less side effects. The first study was conducted in Japanese women who were recently diagnosed to be diabetic [1]. The researchers concluded that though the $\mathrm{HbA1C}$ reduction was more in group receiving $15 \mathrm{mg}$ pioglitazone, there was no statistically significant difference between standard dose (15 $\mathrm{mg}$ ) and low dose group. The study has numerous limitations in addition to being representative of only Japanese females. All the participants were newly diagnosed diabetic with $\mathrm{HbA1C}$ of $7.57 \%$ and $7.69 \%$ in low dose and standard dose groups respectively. The study did not have any control arm on placebo though a similar reduction in $\mathrm{HbA1C}$ can be expected even by lifestyle modification in newly diagnosed diabetics. Pioglitazone being an insulin sensitizers, is not expected to cause hypoglycemia and it is expected that it will not lower $\mathrm{HbA1C}$ further after reaching normoglycemia irrespective of the dose. $\mathrm{HbA} 1 \mathrm{C}$ of $6.96 \%$ was achieved in low dose group after 6 months while that in standard dose arm was $7.0 \%$. Any further lowering in $\mathrm{HbA1C}$ after $6.96 \%$ would be at the cost of hypoglycemia which is not seen with pioglitazone and hence expecting any further lowering by increasing dose of pioglitazone will be unrealistic.

The second study was done in 14 male and 16 female diabetic subjects. All the participants were on sulphonylurea monotherapy at baseline and were additionally given

R. M. Parikh $(\bowtie)$

S K Soni Hospital,

Jaipur, India

e-mail: drrakeshparikh@gmail.com pioglitazone $7.5 \mathrm{mg}$ per day. The authors demonstrated a significant reduction in $\mathrm{HbA} 1 \mathrm{C}$ and significant improvement in serum levels of adiponectin without any significant rise in side effects [2]. The study had very small number of participants and did not have any control arm.

Another study with 80 and 79 subjects being randomized to $7.5 \mathrm{mg}$ and $15 \mathrm{mg}$ of pioglitazone has shown that only standard dose of $15 \mathrm{mg}$ pioglitazone could show significant improvement in HbA1C [3]. A separate study looking at dose response relationship with insulin sensitivity and secretion, has shown no effect of $7.5 \mathrm{mg}$ and $15 \mathrm{mg}$ of pioglitazone on fasting plasma glucose and fasting plasma insulin [4].

Dose calculation is a part of early phase II studies and is the foundation of designing phase III and phase IV studies. The scarce data available from poorly designed studies should not be used to promote use of low dose pioglitazone in the treatment of diabetic subjects.

\section{References}

1. Majima T, Komatsu Y, Doi K, Shigemoto M, Takagi C, Fukao A, et al. Safety and efficacy of low-dose pioglitazone $(7.5 \mathrm{mg} /$ day $)$ vs. standard-dose pioglitazone (15 mg/day) in Japanese women with type 2 diabetes mellitus. Endocr J. 2006;53:325-30.

2. Aso Y, Hara K, Ozeki N, Yatsuka C, Nakano T, Matsumoto S, et al. Low-dose pioglitazone increases serum high molecular weight adiponectin and improves glycemic control in Japanese patients with poorly controlled type 2 diabetes. Diabetes Res Clin Pract. 2009;85:147-52.

3. Aronoff S, Rosenblatt S, Braithwaite S, Egan JW, Mathisen AL, Schneider RL. Pioglitazone hydrochloride monotherapy improves glycemic control in the treatment of patients with type 2 diabetes: a 6month randomized placebo-controlled dose-response study. The Pioglitazone 001 Study Group. Diabetes Care. 2000;23:1605-11.

4. Miyazaki Y, Matsuda M, DeFronzo RA. Dose-response effect of pioglitazone on insulin sensitivity and insulin secretion in type 2 diabetes. Diabetes Care. 2002;25:517-23. 